\title{
Metallentfernungen an der oberen Extremität
}

\author{
Kaywan Izadpanah, Martin Jaeger, Dirk Maier, Norbert P. Südkamp
}

\section{Zusammenfassung}

Die Implantatentfernung im Bereich der oberen Extremitäten sollte bei der Verwendung von modernen Osteosyntheseverfahren nicht als Standardeingriff betrachtet werden. Der überwiegende Anteil moderner Implantate sind anatomisch präformierte Plattensysteme oder im Bereich des Humeruskopfs und des Humerusschafts intramedulläre Kraftträger. Somit ist ein störendes Auftragen der Implantate i.d.R. nicht zu erwarten. Eine Ausnahme bilden hier wohl nur die proximale Ulna und die Klavikula, da der deckende Weichteilmantel bei schlanken Menschen gering ist. Die Indikation zur Implantatentfernung sollte daher kritisch gestellt werden und nur nach entsprechender Indikation, aber auch nach Aufklärung über den zu erwartenden Nutzen für den Patienten und über die verbundenen Risiken erfolgen. Letztere ergeben sich an der oberen Extremität insbesondere aus der häufig kritischen Nähe von Implantaten zu wichtigen Leitungsbahnen, die verletzt zu einer dramatischen Einschränkung der Lebensqualität führen können. Die Indikation zur Metallentfernung sollte daher durch den erfahrenen Orthopäden oder Unfallchirurgen gestellt werden und sich an den geltenden Leitlinien ausrichten. Gleiches gilt für die Durchführung des Eingriffs selbst, insbesondere, da die Entfernung der Implantate häufig mit anderen Eingriffen, z.B. einer Neurolyse oder einer Arthrolyse, kombinert werden muss. Aus dem Schwierigkeitsgrad des additiven Eingriffs, dem Zugangsweg bzw. deren Kombination ergibt sich dann die notwendige Expertise des Operateurs.

\section{Metal Removal in the Upper Extremities}

Implant removal from the upper extremities cannot be considered as a standard procedure. This is especially true for modern implants - such as pre-shaped plates or intramedullary force conductors - as they do not cause changes in skin or other relevant tissues. The proximal ulna and the clavicle are exceptions to this general guideline as these regions have only very little soft tissue coverage. Patients should be well informed about the possible benefits and risks of the procedure. Nonspecific complaints are not likely to improve after implant removal. Special care should be taken during the procedure, as the implants are usually close to the major peripheral nerves, arteries or veins of the upper extremity. Injury to these important structures can severely impair the patient's quality of life. Therefore, implant removal should be indicated by an experienced trauma surgeon in accordance with the AWMF guidelines from the German Society of Trauma Surgery.

\section{Einleitung}

Traditionell sehen viele Patienten die Materialentfernung auch heute noch als den Abschluss einer erfolgreichen operativen Frakturversorgung an. Dies findet auch Ausdruck in der Tatsache, dass Metallentfernungen mit etwa 30\% aller elektiven Eingriffe einen großen Anteil der in Deutschland durchgeführten Operationen darstellen. Dem gegenüber steht, dass der überwiegende Teil moderner Implantate auf den Verbleib im Körper angelegt ist. Die Implantatentfernung muss also nicht erfolgen. Dies wird durch das Implantatdesign, welches ana-

OP-JOURNAL 2016; 32: 120-125

(c) Georg Thieme Verlag KG Stuttgart · New York DOI http://dx.doi.org/10.1055/s-0036-1585258 tomisch präformiert ist und somit wenig oder gar nicht aufträgt, ermöglicht. Zudem zeichnen sich moderne Werkstoffe dadurch aus, dass Effekte wie Implantatkorrosion oder Allergien nicht mehr zu befürchten sind und somit nicht als Indikation für eine Implantatentfernung anzusehen sind. Darüber hinaus entwickeln sich an der oberen Extremität zumeist auch nur geringe biomechanischen Belastungen, sodass implantatassoziierte Änderungen der Knochenbiomechanik, mit Ausnahme der lateralen Klavikula, keine Indikation zur Materialentfernung darstellen.

Trotzdem existieren natürlich auch heute noch Indikationen zur Entfernung von einliegenden Implantaten. In den Leitlinien der Deutschen Gesellschaft für Orthopädie und Unfallchirurgie wurden
Kriterien erarbeitet, die als Entscheidungsgrundlage herangezogen werden sollten.

Die Indikation zur Implantatentfernung sollte im Einklang mit der geltenden AWMF-Leitlinie stehen.

Eine Besonderheit der oberen Extremität ist die Tatsache, dass bei der überwiegenden Mehrheit der Zugänge eine räumlichen Nähe zu wichtigen Leitungsbahnen, wie bspw. zum N. medianus oder zum N. radialis besteht. In der Revision ist immer mit einer veränderten Anatomie zu rechnen, sodass die Materialentfernung nicht - wie weitläufig angesehen - als Anfängereingriff zu betrachten ist. 
An der oberen Extremität ist im Rahmen der Implantatentfernung stets die räumlich Nähe zu den großen Leitungsbahnen zu beachten.

Komplikationen wie die Verletzung dieser Strukturen sind schwerwiegende Komplikationen und können eine Invalidisierung des Patienten nach sich ziehen und sollten daher unbedingt durch den erfahrenen Kollegen zumindest begleitet werden. Dies gilt sowohl für die Indikationsstellung als auch für den Eingriff selber. Hieraus ergibt sich auch die Notwendigkeit einer peniblen Aufklärung über mögliche Risiken eines Eingriffs und den zu erwartenden Nutzen für den Patienten. So muss dem Patienten z.B. verdeutlicht werden, dass durch die Entfernung einer Osteosyntheseplatte bei Entwicklung einer posttraumatischen Arthrose oder einer Humeruskopfnekrose allein keine Linderung seiner Beschwerden zu erwarten ist.

In diesem Artikel sollen Indikationen, allgemein geltende Prinzipien sowie aber auch lokalisationsspezifische Besonderheiten einer Materialentfernung an der oberen Extremität erläutert werden.

\section{Indikationsstellung}

Deutschland ist eines der wenigen Länder, das eine Leitlinie für die Entfernung von Implantaten in der Orthopädie und Unfallchirurgie erstellt hat (Tab. 1). Die vorliegende Arbeit ist daher auch in enger Anlehnung an diese Leitlinie erstellt worden. Bezüglich der Indikation zur Durchführung einer Materialentfernung stellt sie fest, dass diese relativ und selten zwingend ist. Es gibt keine Empfehlung mit hohem Evidenzgrad zur Implantatentfernung. Bei der Indikationsstellung zur Materialentfernung müssen eine Vielzahl von Aspekten in die Betrachtung eingeschlossen werden. Einige Implantate, wie der Fixateur externe oder Kirschner-Drähte, sollten aufgrund der Gefahr der Infektion von Pin-Stellen bzw. der Gefahr sekundärer Dislokation stets entfernt werden [1]. Es müssen also stets die Risiken der Metallentfernung und die Risiken eines Verbleibs des Implantats gegeneinander abgewogen werden.

Risiko der Implantatentfernung und zu erwartender Nutzen für den Patienten müssen gegeneinander abgewogen werden.
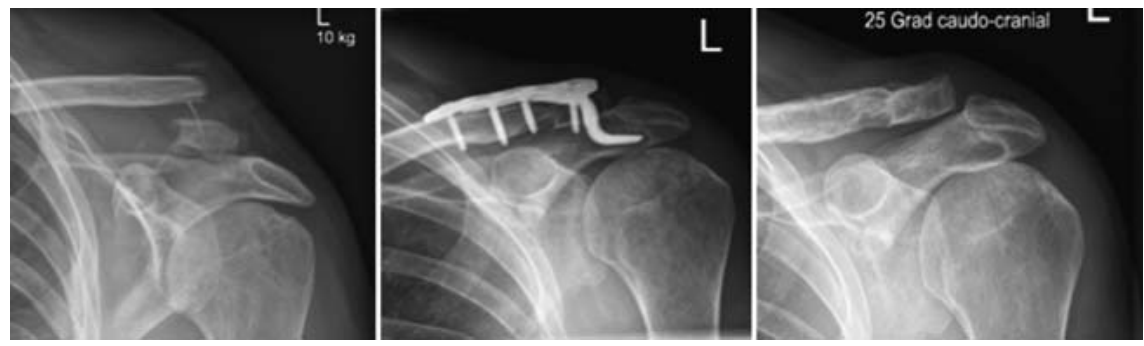

Abb. 1 Indikation zur Entfernung einer Hakenplatte zur Freigabe des Akromioklavikulargelenks nach Osteosynthese einer lateralen Klavikulafraktur.

Die Risiken der Metallentfernung sind im Wesentlichen durch die perioperativen Komplikationen beschrieben, hinzu treten die Risiken, die bei Implantatbrüchen auftreten können. Risiken des Belassens der Implantate entstehen durch die biomechanischen Umbauten am Knochen aufgrund der veränderten Krafteinleitung bzw. Kraftverteilung. Dieses spielt an der oberen Extremität wegen der geringen Belastung jedoch nur eine untergeordnete Rolle. Eine Ausnahme bilden hier Osteosynthesen, die das Akromioklavikulargelenk überbrücken. Hierzu zählt bspw. die Verwendung von Hakenplatten bei der Versorgung lateraler Klavikulafrakturen oder frischer Akromioklavikulargelenksluxationen (Abb.1). Weitere Nachteile von Implantaten können die Einschränkung von Untersuchungsmethoden wie der MRT darstellen. Es hat sich in der Literatur die Einteilung in „klare“ und „diskussionswürdige“ Indikationen etabliert. Sie sind letztlich aber nur als grobe Richtschnur zu werten.

Zu den klaren Indikationen zählen hiernach [1]:

1. störende Implantate

2. Implantate am wachsenden Skelett

3. Metallentfernung aus biomechanischen Gründen

4. Metallentfernung zur Ermöglichung eines Gelenkersatzes

5. Wunsch des Patienten

Zu den diskussionswürdigen Argumenten zählen:

1. älterer Patient

2. topografische Besonderheiten

3. Implantat-Gewebe-Reaktionen

Es existierenen klare und diskussionswürdige Indikationen zur Implantatentfernung.

Es bleibt jedoch i.d.R. eine Einzelfallentscheidung. Beispielsweise bedarf es einer genauen Überprüfung, ob die angegebenen Beschwerden tatsächlich auf das Implantat zurückzuführen sind. Eine Ver- besserung der Beschwerden nach Metallentfernung, gleiches gilt auch für Wetterfühligkeit etc., kann entsprechend der aktuellen Literatur dem Patienten nicht zugesichert werden. Tab. 1 listet allgemein die in der AWMF-Leitlinie genannten Argumente für das Belassen bzw. für die Entfernung eines Implantats auf.

\section{Vorbereitung des Eingriffs}

Nachdem die Indikation zur Materialentfernung gestellt wurde, sollte eine penible Vorbereitung auf den Eingriff erfolgen. Diese beinhaltet zum einen die umfassende Aufklärung des Patienten und außerdem das Treffen der notwendigen logistischen Vorkehrungen. Beides ist notwendig, um den Eingriff erfolgreich durchführen zu können.

\section{Aufklärung}

Die Aufklärung des Patienten sollte umfassend über den zu erwartenden Nutzen und die Risiken des Eingriffs informieren (Tab.2). Es ist Aufgabe des behandelnden Arztes, unspezifische Schmerzen im Bereich des Operationsgebiets von denjenigen eines störenden Implantats, z. B. durch eine Irritation von Sehnen, zu unterscheiden, um den Patienten über das zu erwartende Operationsergebnis aufzuklären. Im Folgenden muss die Aufklärung über mögliche Risiken, wie z.B. Nervenschäden des N. medianus oder des N. radialis bei Operationen im Bereich des Unterarms durchgeführt werden. Zudem müssen sämtliche Zusatzmaßnahmen bzw. erweiterte Eingriffe (Narbenkorrekturen, Neurolysen, Tenolysen, Arthrolysen, Reosteosynthesen, lokale Antibiotikatherapien oder Methylenblau zur intraoperativen Markierung von infiziertem Gewebe bei Fistelbildung) aufgeklärt werden. An gefährdeten Lokalisationen, wie dem Unterarm oder der Klavikula, müssen Patienten über das erhöhte Risiko einer Refraktur informiert werden. Zudem sollten Patienten darüber informiert 
werden, dass ggf. Implantate oder Implantatreste im Körper belassen werden, falls die Entfernung den Aufwand und den damit verbundenen Gewebeschaden nicht rechtfertigen würden. Bei dem Vorliegen einer Infektion bzw. dem dringenden Verdacht auf das Vorliegen einer Infektion sollte eine sterile Einsendung des gesamten Implantats zur mikrobiologischen Untersuchung erfolgen (Sonikation) [2,3]. Dies gilt insbesondere bei der Entfernung von Prothesen.

\section{Logistische Voraussetzungen}

Zur Vorbereitung des Eingriffs ist es zwingend notwendig, sich mit dem Operationsbericht der Implantation auseinanderzusetzen (Tab. 3). Dieser sollte auf Besonderheiten im Rahmen der Implantation und der Lagebeziehung zwischen Nerven und Gefäßen und dem Implantat studiert werden. Außerdem muss vor Durchführung des Eingriffs eine genaue Identifizierung des einliegenden Implantats durchgeführt werden. Dann muss geprüft werden, ob das entsprechende Instrumentarium zur Entfernung des Systems vorhanden ist. Falls nicht, muss dieses zeitgerecht beschafft werden. Außerdem sollten insbesondere zur Entfernung von Titanimplantaten spezielle Instrumentarien vorrätig sein, die zur Entfernung abgebrochener Implantate genutzt werden können.

Das Studium des Operationsberichts der Implantation sowie die Identifikation des einliegenden Implantats stellen eine unabdingbare Grundlage zur Vorbereitung des Eingriffs dar.

\section{Diagnostik}

\section{Klinische Untersuchung}

Im Rahmen der präoperativen klinischen Untersuchung sollte eine gründliche Inspektion des Operationszugangs bzw. der Narbe sowie der umliegenden Weichteilbeschaffenheit erfolgen. Die Hautverhältnisse sollten ebenfalls mit in die Beurteilung einbezogen werden. Im Rahmen dieser Beurteilung muss die Entscheidung getroffen werden, ob die erneute Öffnung der Wunde mit einem erhöhten Risiko der Wundheilungsstörung vergesellschaftet ist bzw. ob eine Korrektur der Narbenverhältnisse möglich oder notwendig ist. Außerdem sollte eine Palpation der Weichteile über den Implantaten bei aktiver und passiver Bewegung erfolgen, um das Gleiten der Weichteile über den Implantaten beurteilen zu kön-

Tab. 1 Allgemeine Argumentation für und gegen eine Materialentfernung (aus der AWMFLeitlinie Implantatentfernung).

\begin{tabular}{lc} 
Belassen der Implantate & Entfernung der Implantate \\
\hline - allgemeine oder lokale Kontraindikatio- & - niedriges Lebensalter \\
nen gegen eine Operation & - Implantate am wachsenden Skelett \\
- fortgeschrittenes Lebensalter & - Bohrdrähte \\
- gefährdete Weichteildeckung & - isolierte Cerclagen am Tibiaschaft \\
- Osteosyntheseplatten am Humerus und & - direkt oder indirekt funktionell störende \\
proximalen Radius (N. radialis) & Implantate \\
- einzelne, nicht störende Schrauben & - temporär gelenkblockierende Implantate \\
- versenkte Gelenkimplantate & (Stellschrauben an der Knöchelgabel, Ha- \\
- nicht störende Cerclagen als Zusatz- & kenplatte am Akromioklavikulargelenk) \\
implantate & - segmentüberbrückende Implantate (Fixa- \\
- Implantate aus Reintitan mit Ausnahme & teur interne) an Brust- und Lendenwirbel- \\
von langen Platten an den unteren Extre- & säule ohne segmentale Fusion, bei Spon- \\
mitäten & dylodese Implantatentfernung nur bei lo- \\
- abgebrochene Metallteile oder verbliebe- & kalen Beschwerden \\
ne Unterlegscheiben nach vorausgegan- & - Verfahrenswechsel bei verzögerter Frak- \\
gener Implantatentfernung & turheilung oder Pseudarthrose \\
- aufwendige Freilegung und zugangs- & - Implantate, bei denen mit einem unphy- \\
bedingte Risiken (Beckenskelett) & siologischen Knochenabbau zu rechnen ist \\
- voluminöse Hüftschraubensysteme in & - Vorbereitung weiterer Maßnahmen im \\
Verbindung mit Marknägeln und Platten & Gelenkbereich \\
beim älteren Patienten & - infizierte Osteosynthesen \\
- ungewöhnlich lange Liegezeit der Implan- & - Wunsch des Patienten \\
tate & \\
&
\end{tabular}

Tab. 2 Checkliste Aufklärung.

- Persistenz von Beschwerden

- Nervenschäden

- Zusatzmaßnahmen (ausführliche Erläuterung)

- Refraktur

- Verbleib von Implantaten bzw. Implantatresten

- allgemeine Operationsrisiken

nen. Außerdem sollten Zeichen einer Allergie bzw. einer Infektion erkannt werden. Zuletzt sollte eine orientierende neurologische Untersuchung erfolgen, um vorbestehende Defizite zu erkennen. Aus der klinischen Untersuchung leiten sich auch die Indikationen zur Durchführung von Begleitoperationen wie Narbenkorrekturen, Arthrolysen, Notwendigkeit einer Reosteosynthese etc. ab.

Eine gründliche klinische Untersuchung muss die vollumfängliche Identifikation etwaiger neurologischer Vorschäden beinhalten.

\section{Präoperative Bildgebung}

Die Standarddiagnostik vor Entfernung von Metallimplantaten ist das Röntgen in 2 Ebenen. Der Fakturbereich, aber auch die Implantate, müssen auf diesen Aufnahmen vollständig abgebildet sein [1]. Insbesondere am Unterarm muss
Tab. 3 Checkliste zur Vorbereitung einer Materialentfernung.

- Nachweis der vollständigen Frakturkonsolidierung

- Lektüre des Operationsberichts (ggf. bestellen)

- Vorhaltung bzw. Bestellung implantatspezifischer Instrumentarien zur Entfernung

- Vorhaltung von Instrumentarien zur Bergung abgebrochener Implantate

der Frakturbereich in 2 senkrecht aufeinander stehenden Ebenen (nicht die Osteosyntheseplatten) dargestellt werden. Nur so ist es möglich, die vollständige Konsolidierung der Fraktur nachzuweisen. Neben der Beurteilung der Konsolidierung ist auf diesen Röntgenbildern die Untersuchung des Implantats bzw. der Implantate durchzuführen. Es gilt, Lockerungen oder Implantatbrüche zu erkennen. Diese können zum einen Hinweise auf eine unvollständige Durchbauung geben, darüber hinaus können aber auch Komplikationen bei der Materialentfernung antizipiert bzw. zu verbleibendes Implantatmaterial präoperativ identifiziert werden.

In speziellen Fragestellungen, d.h. bei zweifelhafter Konsolidierung, sollte zusätzlich eine Computertomografie erfolgen. In seltenen Fällen kann darüber hinaus eine SPECT-CT erfolgen, um bspw. einen Zusammenhang zwischen einzel- 

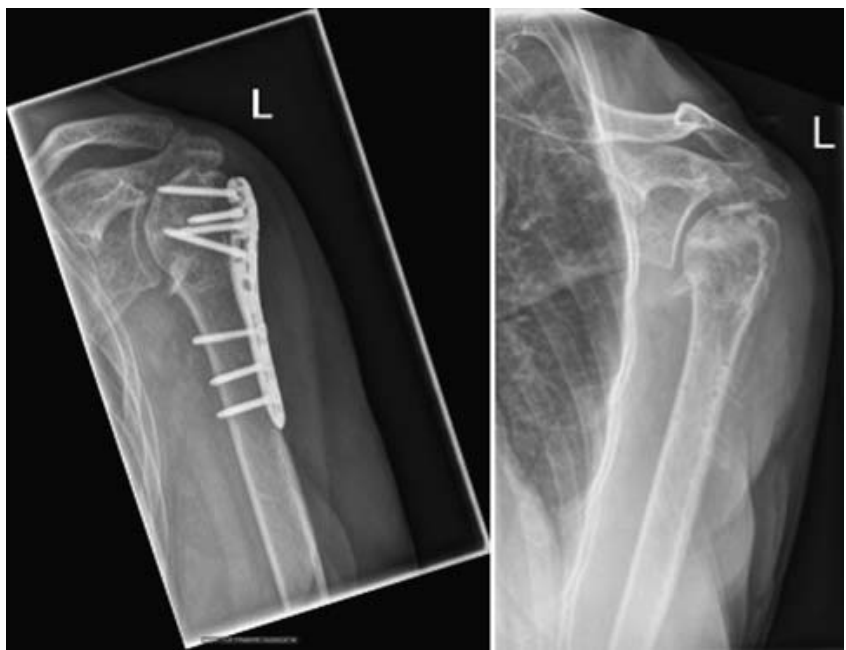

Abb. 2 Indikation zur Plattenentfernung bei sekundär überstehender Schraube bei posttraumatischer, avaskulärer Humeruskopfnekrose.

nen freien Schrauben und Gelenkschmerzen bzw. der Feststellung einer Osteomyelitis zu ermöglichen [4]. Diese Untersuchungsmethode ist jedoch nicht Teil der Leitlinien.

Das Röntgen in 2 Ebenen mit Darstellung der Frakturkonsolidierung ist eine Voraussetzung der Implantatentfernung.

\section{Zeitpunkt der Implantatentfernung}

Grundsätzlich besteht bei komplikationsloser Knochenheilung und ausbleibendem Infekt die Indikation zur Materialentfernung in gelenknahen Skelettabschnitten nach ca. 9-12 Monaten, in diaphysären Gelenkabschnitten nach 18-24 Monaten (bei winkelstabilen Implantaten aus Titan auch schon nach 12 Monaten) und am distalen Radius nach 3-6 Monaten [5]. Ausnahmen stellen Infektionen dar, bei denen nach der 2. Revision keine Kontrolle des Infekts erreicht werden konnte. Hier sollte, wenn immer möglich, im Rahmen der 2. Revision eine Implantatentfernung bzw. ein Implantatwechsel erfolgen.

\section{Topografische Besonderheiten}

Wenige retrospektive Studien existieren, die eine systematische Untersuchung von Materialentfernungen an der oberen Extremität untersucht haben. Beschwerden von Patienten sind in der Mehrheit die Indikation zur Durchführung des Eingriffs [6]. Mehrheitlich war das Auftreten von postoperativen Komplikationen Endpunkt der Untersuchung und nur 2 Studien befassten sich mit den Indikationen, die zur Materialentfernung führten. Über die Arbeiten und die Lokalisationen hinweg resümierten die Autoren, dass eine routinemäßige Entfernung nicht erfolgen sollte und wenn doch, dann nur durch den erfahrenen Chirurgen.

\section{Metallentfernung an den exponierten Regionen Klavikula und proximale Ulna}

Störende Implantate sind eine der häufigsten Ursachen für die Entfernung von Osteosynthesematerialien [1]. Aufgrund der geringen Weichteildeckung im Bereich des Klavikulaschafts klagen Patienten häufig über eine Irritation durch die Osteosyntheseplatte beim Tragen von Taschen oder Rucksäcken. Gleiches gilt für die proximale Ulna, da es beim Abstützen auf den Ellenbogen zu Weichteilirritationen kommen kann. Die Indikation zur Entfernung der Osteosyntheseplatte sollte daher eng gestellt werden. Eine Entfernung dieser Implantate kann ab dem 6. postoperativen Monat erfolgen. Es muss jedoch auf eine strenge Kontrolle der radiologischen Konsolidierung der Klavikula- bzw. Ulnafraktur geachtet werden. Refrakturraten von ca. 2-7\% werden nach Entfernung von Plattenosteosynthesen an der Klavikula beschrieben [7]. In der Nachbehandlung kann jeweils eine funktionelle Belastung erfolgen. Kontaktsportarten sind jedoch erst 12 Wochen nach Plattenentfernung zu empfehlen, da durch die Schraubenlöcher das Frakturrisiko bis zu deren Durchbauung erhöht ist.

\section{Metallentfernung aus biomechanischen Gründen an der lateralen Klavikula}

Mit der Hakenplatte osteosynthetisch versorgte Luxationen des Akromioklavikulargelenks und der lateralen Klavikulafraktur stellen aufgrund der biomechanischen Beanspruchung eine absolute Operationsindikation dar. Physiologisch kommt es bei Abduktion der Schulter über die Horizontale zunehmend $\mathrm{zu}$ einer Rotation der Klavikula sowie einem Dorsalwandern der lateralen Klavikula im Verhältnis zum Akromion. Da dieses physiologische Gelenkspiel blockiert wird, sollte die Hakenplatte wieder entfernt werden. Eine Entfernung erfolgt nach Konsolidierung der lateralen Klavikula, ca. 3-6 Monate postoperativ. Nach Behandlung einer Akromioklavikulargelenksluxation sollte die Metallentfernung nach 3 Monaten erfolgen.

\section{Metallentfernung am proximalen Humerus}

Die zunehmende Verwendung moderner winkelstabiler Plattensysteme und moderner Marknägel bei der Versorgung von proximalen Humerusfrakturen hat zu einer deutlichen Steigerung der Versorgungsqualität geführt [8]. Gleichwohl werden in der aktuellen Literatur Komplikationsraten, die eine operative Revision notwendig machen, von bis zu 36\% angegeben $[9,10]$. Hierzu zählen bspw. Humeruskopfnekrosen mit sekundärer Fraktursinterung oder primäre Implantatfehllagen (Abb. 2). Jost und Mitarbeiter konnten in einer retrospektiven Analyse von 121 Patienten, die mittels einer winkelstabilen Plattenosteosynthese versorgt worden sind, feststellen, dass partielle Metallentfernungen im Bereich das proximalen Humerus häufig nicht zielführend sind. In ihrer Population mussten 13 von 16 Patienten (81\%) zumindest eine weitere operative Maßnahme über sich ergehen lassen. Dies war entweder die vollständige Implantatentfernung oder ein endoprothetischer Ersatz des Gelenks (53\%). Somit können partielle Metallentfernungen am proximalen Humerus nicht empfohlen werden. In dieser Studie wurde bei 41 Patienten (33\%) primär eine komplette Metallentfernung durchgeführt. Dies aufgrund von avaskulären Humeruskopfnekrosen.

Aus der Erfahrung der Autoren gibt es auch einen Anteil von Patienten mit einer in anatomischer Stellung konsolidierten Fraktur ohne Nachweis von Humeruskopfnekrosen, die eine endgradige Bewegungseinschränkung aufweisen, die auch durch intensive Physiotherapie nicht zu beheben ist. Bei jungen Patienten mit hohem Funktionsanspruch kann hier die Implantatentfernung in Kombination mit einer Arthrolyse des Subakromialraums zu einer Beseitigung der Bewegungseinschränkung führen. 
Tab. 4 Tabellarische Zusammenfassung von Indikation, Zeitpunkt der Entfernung, perioperative Risiken und die Nachbehandlung bei Metallentfernungen entsprechend der anatomischen Region.

\begin{tabular}{lllll} 
Lokalisation & Indikation & $\begin{array}{l}\text { Zeitpunkt der } \\
\text { Entfernung }\end{array}$ & $\begin{array}{l}\text { spezifische } \\
\text { Risiken }\end{array}$ & $\begin{array}{l}\text { Nachbehand- } \\
\text { lung }\end{array}$ \\
\hline Klavikula & $\begin{array}{l}\text { störendes bzw. } \\
\text { auftragendes } \\
\text { Implantat }\end{array}$ & $\begin{array}{l}\text { nach Konsoli- } \\
\text { dierung }\end{array}$ & $\begin{array}{l}\text { Verletzung } \\
\text { der Nn. }\end{array}$ & $\begin{array}{l}\text { funktionell, } \\
\text { Kontaktsport } \\
\text { nach 3 Monaten }\end{array}$ \\
\hline $\begin{array}{l}\text { proximaler } \\
\text { Humerus }\end{array}$ & $\begin{array}{l}\text { Humeruskopfne- } \\
\text { krose mit Schrau- } \\
\text { benperforation }\end{array}$ & 9-12 Monate & $\begin{array}{l}\text { Verletzung } \\
\text { N. axilaris }\end{array}$ & $\begin{array}{l}\text { funktionell, } \\
\text { Kontaktsport } \\
\text { nach 3 Monaten }\end{array}$ \\
\hline $\begin{array}{l}\text { Humerus- } \\
\text { schaft }\end{array}$ & $\begin{array}{l}\text { Infektionen, } \\
\text { Pseudarthrosen }\end{array}$ & individuell & $\begin{array}{l}\text { Verletzung } \\
\text { des N. radialis }\end{array}$ & individuell \\
\hline $\begin{array}{l}\text { proximale } \\
\text { Ulna }\end{array}$ & $\begin{array}{l}\text { störendes bzw. } \\
\text { auftragendes }\end{array}$ & nach Konsoli- \\
Implantat & dierung & $\begin{array}{l}\text { Verletzung } \\
\text { des N. ulnaris }\end{array}$ & $\begin{array}{l}\text { funktionell, } \\
\text { Kontaktsport } \\
\text { nach 3 Monaten }\end{array}$ \\
\hline Unterarm & $\begin{array}{l}\text { junger Patient nach } \\
\text { Konsolidierung, } \\
\text { Einschränkung der } \\
\text { Umwendbewegung }\end{array}$ & 9-15 Monate & $\begin{array}{l}\text { Verletzung } \\
\text { des N. radialis }\end{array}$ & $\begin{array}{l}\text { funktionell, } \\
\text { Kontaktsport } \\
\text { nach 6 Monaten }\end{array}$ \\
& $\begin{array}{l}\text { junger Patient nach } \\
\text { Konsolidierung, } \\
\text { Tendovaginitiden }\end{array}$ & $\begin{array}{l}\text { nach Konsoli- } \\
\text { dierung }\end{array}$ & $\begin{array}{l}\text { Verletzung } \\
\text { N. medianus }\end{array}$ & $\begin{array}{l}\text { funktionell, } \\
\text { Kontaktsport } \\
\text { nach 3 Monaten }\end{array}$ \\
\hline distaler Radius & & &
\end{tabular}

Letztere resultiert zumeist aus einer Verklebung der Verschiebeschichten. Hierfür ist das Titanimplantat maßgebend, denn die raue Oberfläche induziert eine starke Zell- bzw. Gewebeadhärenz. Auch hierbei handelt es sich um einen hochelektiven Eingriff, der mit dem Patienten ausführlich besprochen werden muss. Die Verwendung von Stahlimplantaten bei jüngeren Patienten kann diese Problematik in der Häufigkeit des Auftretens reduzieren.

Hora und Mitarbeiter [11] berichteten in einer Studie von Komplikationen nach Marknagelentfernungen aus dem Humerus. Es wurden 22 Patienten in die retrospektive Analyse eingeschlossen. Hier kam es bei 28,6\% der Fälle zu Komplikationen. Diese bestanden in der Mehrheit in oberflächlichen Wundinfektionen oder persistierenden Schmerzen an der Nageleintrittstelle.

\section{Metallentfernung am Humerusschaft und dem distalen Humerus}

Die Metallentfernung nach osteosynthetischer Versorgung von Humerusschaftfrakturen ist nach Dafürhalten der Autoren nach Versorgung mittels Osteosyntheseplatte nur im Falle von Komplikationen, wie dem Auftreten von Infektionen oder der Entwicklung einer Pseudarthrose, indiziert. Aufgrund des hohen Risikos der Verletzung des N. radialis kann eine routinemäßige Indika- tion bzw. die Entfernung aufgrund des Patientenwunschs nicht empfohlen werden. Besteht die Indikation zur Materialentfernung aufgrund der o.g. Komplikationen, muss ein sehr genaues Studium des Operationsberichts erfolgen, um die Lagebeziehung zwischen Osteosyntheseplatte und dem $\mathrm{N}$. radialis genau zu erfahren. Das Aufsuchen des N. radialis sowie die Präparation ist daher essenziell, um eine Verletzung zu vermeiden. Die Nachbehandlung kann funktionell erfolgen. Kontaktsportarten sollten erst 3 Monate postoperativ angefangen werden.

\section{Metallentfernung am Unterarm}

Der Unterarm zählt zu den verhältnismäßig gut untersuchten Regionen der oberen Extremitäten in Bezug auf Komplikationen durch die Materialentfernungen. Das Auftreten von Refrakturen wurde von unterschiedlichen Autoren zwischen 4 und 9\% angegeben, sodass eine allgemeine Empfehlung zur Metallentfernung bei asymptomatischen Patienten nicht gestellt werden kann [6, 12]. Die Indikation zur Materialentfernung besteht bei störenden Implantaten, insbesondere, wenn diese eine freie Umwendbewegung des Unterarms verhindern bzw. im Bereich der distalen Ulna auftragen. Das erhöhte Refrakturisiko besteht zumindest innerhalb des 1. Jahres. Somit sollten nach Materialentfernung in diesem Zeitraum keine Kontakt- sportarten durchgeführt werden. Platten im Bereich des proximalen Radius sollten aufgrund der topografischen Beziehung zum Ramus profundus n. radialis nur in Ausnahmesituationen entfernt werden [1].

\section{Metallentfernung am distalen Radius}

Der distale Radius wird auch heute noch bei sehr distalen und besonderen Frakturmorphologien mit einem Fixateur externe und/oder Kirschner-Drähten ausbehandelt. Diese Implantate müssen selbstverständlich nach Frakturkonsolidierung entfernt werden. Die Eingriffe erfolgen normalerweise in Lokalanästhesie (K-Drähte) oder ohne Betäubung (Fixateur). Obgleich moderne Plattensysteme heute nur noch geringe Plattendicken aufweisen und anatomisch präformiert sind, können sie, wenn sie nicht weichteilig gedeckt sind, zur Irritation und im Verlauf zur Ruptur von Sehnen führen (selbst bei volarer Plattenlage). Besondere Gefährdung besteht am M. extensor pollicis longus oder dem M. flexor pollicis longus. Die Diagnosestellung von überstehenden Schrauben bzw. die enge Lagebezieung von Platte und Sehne kann gut im Ultraschall dargestellt werden. Bei asymptomatischen Patienten sollten die Osteosyntheseplatten belassen werden. Nach erfolgreicher Entfernung kann innerhalb von 3 Monaten eine Vollbelastung mit Kontaktsport angefangen werden, da das Refrakturrisiko gering ist [13].

\section{Literatur}

${ }^{1}$ Müller-Färber J. Die Metallentfernung in der Unfallchirurgie. Unfallchirurg 2003; 106: 653-668

2 Dresing K. Infektionen in Unfallchirurgie und Orthopädie. Oper Orthop Traumatol 2013; 25: 220-224

${ }^{3}$ Esteban J, Alonso-Rodriguez N, del-Prado G et al. PCR-hybridization after sonication improves diagnosis of implant-related infection. Acta Orthop 2012; 83: 299-304

${ }^{4}$ Huellner MW, Strobel K. Clinical applications of SPECT/CT in imaging the extremities. Eur J Nucl Med Mol Imaging 2014; 41 (Suppl. 1): S50-S58

${ }^{5}$ Kirchner $R$. Metallentfernung. In: Jauch KW, Mutschler W, Hoffmann JN, Kanz KG, Hrsg. Chirurgie Basisweiterbildung. Berlin, Heidelberg: Springer; 2013: 216-221

${ }^{6}$ Vos DI, Verhofstad MHJ. Indications for implant removal after fracture healing: a review of the literature. Eur J Trauma Emerg Surg 2013; 39: 327-337

7 Shin SJ, Do NH, Jang KY. Risk factors for postoperative complications of displaced clavicular midshaft fractures. J Trauma Acute Care Surg 2012; 72: 1046-1050

${ }^{8}$ Konrad G, Hirschmüller A, Audigé L et al. Comparison of two different locking plates for two-, three- and four-part proximal humeral 
fractures - results of an international multicentre study. Int Orthop 2011; 36: 1051 1058

${ }^{9}$ Jost B, Spross C, Grehn $\mathrm{H}$ et al. Locking plate fixation of fractures of the proximal humerus: analysis of complications, revision strategies and outcome. J Shoulder Elbow Surg 2013; 22: 542-549

10 Konrad G, Audigé L, Lambert $S$ et al. Similar outcomes for nail versus plate fixation of three-part proximal humeral fractures. Clin Orthop Relat Res 2011; 470: 602-609
${ }^{11}$ Hora K, Vorderwinkler KP, Vécsei V et al. [Intramedullary nail removal in the upper and lower limbs. Should we recommend this operation?]. Unfallchirurg 2008; 111: 599601, 603-605

12 Rumball K, Finnegan M. Refractures after forearm plate removal. J Orthop Trauma 1990; 4: 124-129

${ }^{13}$ Krettek PC, Müller C, Meller $R$ et al. Ist eine routinemäßige Implantatentfernung nach unfallchirurgischen Eingriffen sinnvoll? Unfallchirurg 2012; 115: 315-322
PD Dr. med. Kaywan Izadpanah, MD

Oberarzt

Dr. med. Martin Jaeger, MD

Oberarzt

PD Dr. med. Dirk Maier, MD

Oberarzt

Prof. Dr. med. Norbert P. Südkamp

Ärztlicher Direktor

Department Chirurgie

Klinik für Orthopädie und

Unfallchirurgie

Hugstetter Straße 55

79106 Freiburg

kaywan.izadpanah@

uniklinik-freiburg.de 